\title{
Characteristics of Steady and Transient Superfluid Transport for the Cooling of Superconducting Rotating Machines
}

\author{
Thomas C. Chuang \\ NEO Research Center, Van Nung University of Science and Technology, Jung-Li, Taiwan, \\ Republic of China
}

The unique properties of superfluid helium (He II) make it a very efficient cooling agent for superconducting rotating machines. Steady and transient transport characteristics and design formulas for the cooling of superconducting windings are enumerated in this article. Several superfluid transport analytical models and useful design equations are discussed: laminar flow; turbulent flow; and pure superfluid flow under steady-state and transient conditions. An effort was made to consolidate all analytical models and experimental results into a common framework. Under conditions of steady He II transport, a dimensionless heat flux number $N_{q}$, a dimensionless driving force number $N_{\nabla T}$, and a characteristic length where used so that a generalized equation could be derived to describe superfluid transport in any geometry. In the case of transient transport of He II, a dimensionless heat flux number $N_{q *}$ and a dimensionless driving time number $N_{t}$ were used so that a generalized equation could be derived to describe transient superfluid transport in laminar flow and turbulent regimes. Many experimental data were compiled to substantiate the analysis.

Keywords Kapitza conductance, Porous plugs, Superfluid transient transport, Superfluid transport

Large-scale superconducting magnets are expected to play a major role in future energy systems (Aized et al., 1999; Brechna,

Received 1 April 2003; accepted 10 June 2003.

The author expresses special thanks to Professor T. H. K. Frederking (Chemical Engineering Department, University of California, Los Angeles) for his constant encouragement and support until the day he left us in 2001. The author is also indebted to input from Dr. S. W. K. Yuan.

Address correspondence to Thomas C. Chuang, NEO Research Center, Van Nung University of Science and Technology, 1, Van Nung Road, Jung-Li, Taiwan, ROC. E-mail: tomchuang@cc.vit.edu.tw

1973; Hull et al., 1999; Leupold et al., 1988; Miller et al., 1994; Singh et al., 1999; Turk, 1989; Vajda et al., 1999). Figure 1 shows the rotor and stator assembly in a conceptual design of a superconducting generator. Aized (1999) described the development of a $1000 \mathrm{hp}$ superconducting motor in which the field winding is made of a superconductor that is cooled by means of closed-cycle helium refrigeration. The stator winding is watercooled by embedded cooling tubes. Vajda (1999) demonstrated the design and construction of an experimental superconducting synchronous machine. It has a permanent magnet rotor and a superconducting stator. Magnets of this type permit the construction of lightweight motors and generators that have obvious advantages for marine and airborne use. There is growing interest in using a superfluid as the coolant in superconducting rotating machines like superconducting motors and generators because of its unique and favorable cooling characteristics. Phenomena that tie directly into the safety and reliability of such superconducting windings are characterized by the thermodynamics of quasi-steady heat transfer and transient heat transfer to liquid helium (He II) during superconductor-coolant interaction.

Superfluid heat transport presents many advantages: (1) Higher thermal stability due to lower operating temperatures; (2) higher heat conductivity, which reduces the generation of unfavorable vapor bubbles between windings; (3) insensitivity to winding orientation, which allows for flexibility in the geometry of the winding design. These advantages must be balanced against the added refrigeration requirement and the higher cost as compared with other cooling systems. For example, Kovalev (1998) reported two types of high-temperature superconducting alternators cooled by liquid hydrogen.

Many investigators have compiled data concerning the cooling of superconducting windings with He II; however, it appears that there is no consistent and universal means of evaluating data in a common framework. This article presents the use of dimensionless numbers for the heat flux density, the thermomechanical driving force, the mechanical driving force, and the transient time associated with the He II transport so that a generalized 


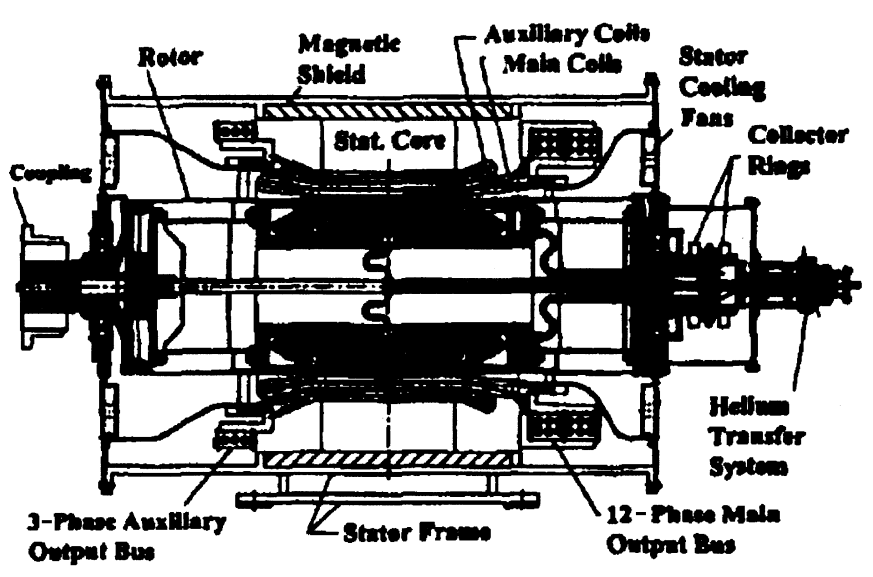

FIGURE 1

A conceptual design of a superconducting generator. (From Singh et al., 1999).

relationship among these numbers can be derived. Various data from a number of investigators are then used to reinforce the relationships. These generalized relationships are useful for providing good estimates of system design associated with He II transport.

Two general heat transport geometries are considered-the surface transport and the duct transport. The surface transport simulates the cooling of a superconducting winding in a pool of superfluid, whereas the duct transport simulates heat removal from fine gaps between superconductor windings. A first-order estimate of the steady-state thermal resistance and the characteristics of the dynamic quench of superconductors can be derived for a given winding geometry.

\section{He II STEADY-STATE HEAT AND MASS TRANSFER}

\section{Surface He II Transport: Kapitza Contact Resistance}

Theoretically, there are two major cases of superconductorliquid interaction. First, in the temperature $(T)$ range below $0.6 \mathrm{~K}$, phonons are the dominant entropy carriers, and interaction between the phonons of the superconductor and the liquid leads to high resistance caused by the mismatch in the sound speeds of the superconductor and the liquid. Second, in the temperature range above $1 \mathrm{~K}$, where roton states are occupied, the rotational entropy carriers contribute more significantly to the thermal properties of He II than do the phonons. In the first case, the theory of Khalatnikov (1952) describes the exchange of energy between the solid and the He II by radiation of sound waves. The thermal resistance caused by the acoustic mismatch is found to be proportional to $T^{-3}$. At $T>1 \mathrm{~K}$, the numerical results of the theory no longer agree with experimental data; nevertheless, the $T^{3}$ - law for the thermal Kapitza conductance $h$ still appears to be a reasonable approximation. Kapitza conductance is a property essential to nonboiling heat transfer from metal to He II. Temperature discontinuity occurs at the superconductor-
He II interface. For a small temperature difference, $\Delta T \ll T$, the Kapitza conductance may be written as

$$
h_{o}=\lim _{\Delta T \approx 0}(q / \Delta T)
$$

where $q$ is the heat flux density. According to a simplified picture of longitudinal sound quanta (longitudinal, compressional photons), for a general transverse and longitudinal phonon system striking the interface between a superconductor and He II, it is the superconductor's phonon system that is the primary contributor to resistance to heat transfer.

Yuan and colleagues (1989) showed that the heat flux density of the "black-body Debye phonon cavity radiation" could be abbreviated as

$$
q=\sigma_{B}\left[(T+\Delta T)^{4}-T^{4}\right]
$$

where $\sigma_{B}$ is the phonon radiation constant of the superconductor. Combining Equations (1) and (2) gives

$$
\begin{aligned}
h_{o} & =4 \sigma_{B} T^{3} \\
h & =h_{o}\left[1+1.5(\Delta T / T)+(\Delta T / T)^{2}+0.25(\Delta T / T)^{3}\right] .
\end{aligned}
$$

Chuang (1981) measured the Kapitza conductance of niobium-titanium-copper superconductor immersed in He II; the critical temperature of the superconductor was around $10 \mathrm{~K}$. Figure 2 shows the results, which are in good agreement with the theory. Iwamoto and colleagues (2001) found that the surface finish, the degree of oxidation of the surface, and the material in the coating of the specimen being tested all play a very important role in the magnitude of the Kapitza conductance.

For practical applications, Kapitza conductance can be extended to the He I regime. It was found that the Kapitza conductance in He I can be estimated based on the Kapitza conductance

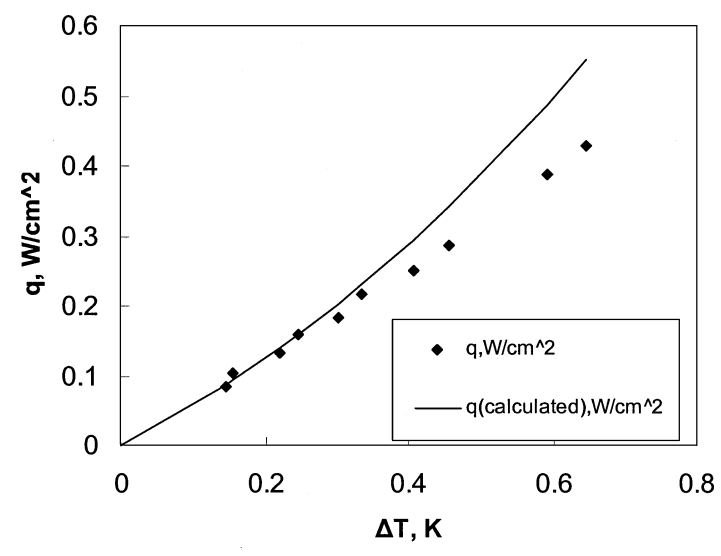

FIGURE 2

Kapitza conductance at $\Delta T \sim 0$ is equal to $h_{o}=.0628 T^{3}$ $\mathrm{W} / \mathrm{Kcm}^{2}$ for a niobium-titanium-copper superconductor $\left(T_{b}=\right.$ $2.05 \mathrm{~K}, P=P_{\text {sat }}$ ). (Data points from Chuang, 1981). 
value for the same surface in He II through the $T^{3}$ dependence. Shiotsu and colleagues (1996) found that the Kapitza conductance is independent of the pressure over the He II.

\section{Heat Transport Through Ducts in He II}

A simplified heat transport model can be constructed to analyze the heat transport between superconductor windings. It is assumed that many fine ducts are formed between superconductor windings when heat is supplied at the bottom of the duct. Superfluid, unlike ordinary fluid, presents the unusual characteristic of removing heat from these ducts so efficiently that ordinary formulas for fluid mechanics cannot adequately describe it. A two-fluid model was developed to explain the unique behavior of the quantum fluid.

It was Tisza (1940) who first recognized the possibility of evading the pitfalls of a rigorous molecular-kinetic theory by employing the qualitative properties of a degenerating BoseEinstein gas to develop a consistent macroscopic theory, referred to as the two-fluid model. In most transport situations, He II heat transport occurs by means of convection of the entropy carrier system, called normal fluid based on a phenomenological twofluid model.

The two-fluid theory is based on the assumptions that He II consists of two mutually interpenetrating fluids, the "superfluid" of density $\rho_{s}$ and the "normal fluid" of density $\rho_{n}$. Each of them is supposed to have its own velocity field, $v_{s}$ and $v_{n}$, respectively. The superfluid corresponds to the condensed phase of the Base-Einstein system. Accordingly, it does not contribute to the entropy. The normal fluid is supposed to be the carrier of the excitations (photons an rotons) of the liquid. Aside from this, it is assumed that the viscosity coefficient of the superfluid is zero. The density is given as

$$
\rho=\rho_{s}+\rho_{n}
$$

and momentum of the fluid in motion is

$$
\begin{aligned}
& \vec{J}=\rho_{s} \cdot \overrightarrow{v_{s}}+\rho_{n} \cdot \overrightarrow{v_{n}} .
\end{aligned}
$$

Because the superfluid has zero entropy, heat transport through $\mathrm{He}$ II is accomplished by the motion of the normal fluid. It follows directly that the heat flux density $q$ is given by

$$
q=\rho S T v_{n} .
$$

If, in addition, there is no net mass flow of He II, there is the constant

$$
\left\langle\rho_{s} \cdot v_{s}+\rho_{n} \cdot v_{n}\right\rangle=0
$$

where the brackets denote an average over the cross-section perpendicular to the heat flow. Physically, this corresponds to the motion of "warm" normal fluid away from the heat source and the counterflow motion of "cold" superfluid toward the heat source.
The commonly accepted hydrodynamic equations for the two components are modifications of the classical Navier-Stokes equation, as described by Schlichting (1960):

$$
\begin{aligned}
& \rho_{n} \frac{\partial v_{n}}{\partial t}+\rho_{n} v_{n} \times \nabla v_{n} \\
& \quad=-\frac{\rho_{n}}{\rho} \nabla p-\rho_{s} S \nabla T+\eta \nabla^{2} v_{n}+F_{s n}
\end{aligned}
$$

and

$$
\rho_{s} \frac{\partial v_{s}}{\partial t}+\rho_{s} v_{s} \times \nabla v_{s}=-\frac{\rho_{s}}{\rho} \nabla p+\rho_{s} S \nabla T-F_{s n}
$$

where $p, S$, and $\eta$ are, respectively, the pressure, entropy, and (normal fluid) viscosity, and $F_{s n}$ is a sometimes present mutual friction between the $s$ and $n$ components.

\section{Small Thermal Flux: Laminar Flow}

As long as the velocities $v_{s}$ and $v_{n}$ are relatively small (corresponding to a low heat flux), the frictional force $F_{s n}$ and the acceleration terms $d v / d t$ are zero (assuming steady state), with the result

$$
\nabla p=\rho S T v_{n}
$$

from Equation (9). Equation (10) is known as the London equation. This pressure gradient causes laminar flow to the normal fluid in accordance with the classical Poiseuille equation

$$
\nabla p=\eta \nabla^{2} v_{n}
$$

which is obtained by adding Equations (8) and (9). The solution to this equation for a long tube of diameter $d$ is

$$
\nabla p=\frac{32 \eta v_{n}}{d^{2}}
$$

which, when combined with Equations (6) and (10), and by replacing $d$ with $L_{c}$ as the generalized term, gives

$$
q=L_{c}^{2} \frac{\rho^{2} S^{2} T}{32 \eta} \nabla T
$$

where $L_{c}=D /\left(32^{0.5}\right)$ is for capillaries; $L_{c}=\left(K_{p}^{0.5}\right)$ is for porous media (Darcy's law); and $L_{c}=D /\left(12^{0.5}\right)$ represents the transport in slits, as shown by Yuan and colleagues (1990).

It is convenient to introduce a dimensionless Reynolds number equivalent number $N_{q}$ and a Rayleigh number equivalent number $N_{\nabla T}$, where

$$
N_{q}=\frac{\rho v_{n} L_{c}}{\eta_{n}}=\frac{q L_{c}}{\eta_{n} S T}
$$

and

$$
N_{\nabla T}=\frac{\rho \nabla p L_{c}^{3}}{\eta_{n}^{2}}=\frac{\rho^{2} S T L_{c}^{3}}{\eta_{n}^{2}} .
$$


Combining Equations (13), (14), and (15), a simple dimensionless equation can be used to describe laminar flow in many different He II flow geometries:

$$
N_{q}=N_{\nabla T}
$$

\section{Large Thermal Flux: Turbulent Transport}

If the superfluid and normal fluid components move at high speed with each other, a mutual friction appears in the counterflow of superfluid and normal fluid. In a turbulent regime, the hydrodynamic Equations (8) and (9) appear to be dominated by the mutual friction term $F_{s n}$ per unit volume. Gorter and Mellink (1949) showed that experiments on fountain pressures and thermal conduction are consistent with

$$
F_{s n}=A_{\mathrm{GM}} \rho_{s} \rho_{n}\left|v_{s}-v_{n}\right|^{3}
$$

where the constant $A_{\mathrm{GM}}$ is usually called the Gorter-Mellink constant. This is a slowly varying function of the temperature of He II. The thermal gradient in the presence of mutual friction is found by neglecting the acceleration term on the left-hand side of Equation (8) along with the $\nabla p$ term and the viscous term $\eta \nabla^{2} v_{n}$, with the result

$$
\nabla T=\frac{F_{s n}}{\rho S_{s}}
$$

When combined with Equations (6), (7), and (17), this gives

$$
\nabla T=\frac{A_{\mathrm{GM}} \rho_{n}}{S}\left(\frac{q}{\rho_{s} S T}\right)^{3} .
$$

Dimensionless analysis of the heat transport through He II-filled ducts was carried out by Soloski and Frederking (1980).

$$
A_{\mathrm{GM}}=\operatorname{const}\left(\rho / \rho_{s}\right) / \eta_{n}
$$

where const $=1 / K_{\mathrm{GM}}^{3}$ (also called the Gorter-Mellink constant). Figure 3 shows that by comparing the experimental data for $A_{\mathrm{GM}}$ and Equation (20), Soloski and Frederking (1980) found that $K_{\mathrm{GM}}=11.3$ exhibited a good fit at temperatures from $1.3 \mathrm{~K}$ to $2.1 \mathrm{~K}$.

Yuan et al. (1990) showed a dimensionless form as

$$
\left(\rho / \rho_{s}\right) N_{q}=K_{\mathrm{GM}}\left[\left(\rho_{s} / \rho_{n}\right) N_{\nabla T}\right]^{1 / 3} .
$$

Figure 4 demonstrates that for wider ducts, Equation (21) is universal to describe many experimental results in the literature.

The heat flux density can now be calculated by combining Equations (19) and (20) and by looking up the thermodynamic properties at a given temperature and pressure for a specified temperature gradient:

$$
q=K_{\mathrm{GM}} \eta_{n} S T \frac{\rho_{s}}{\rho}\left(\frac{\rho^{2} \rho_{s} S \nabla T}{\rho_{n} \eta_{n}^{2}}\right)^{1 / 3}
$$

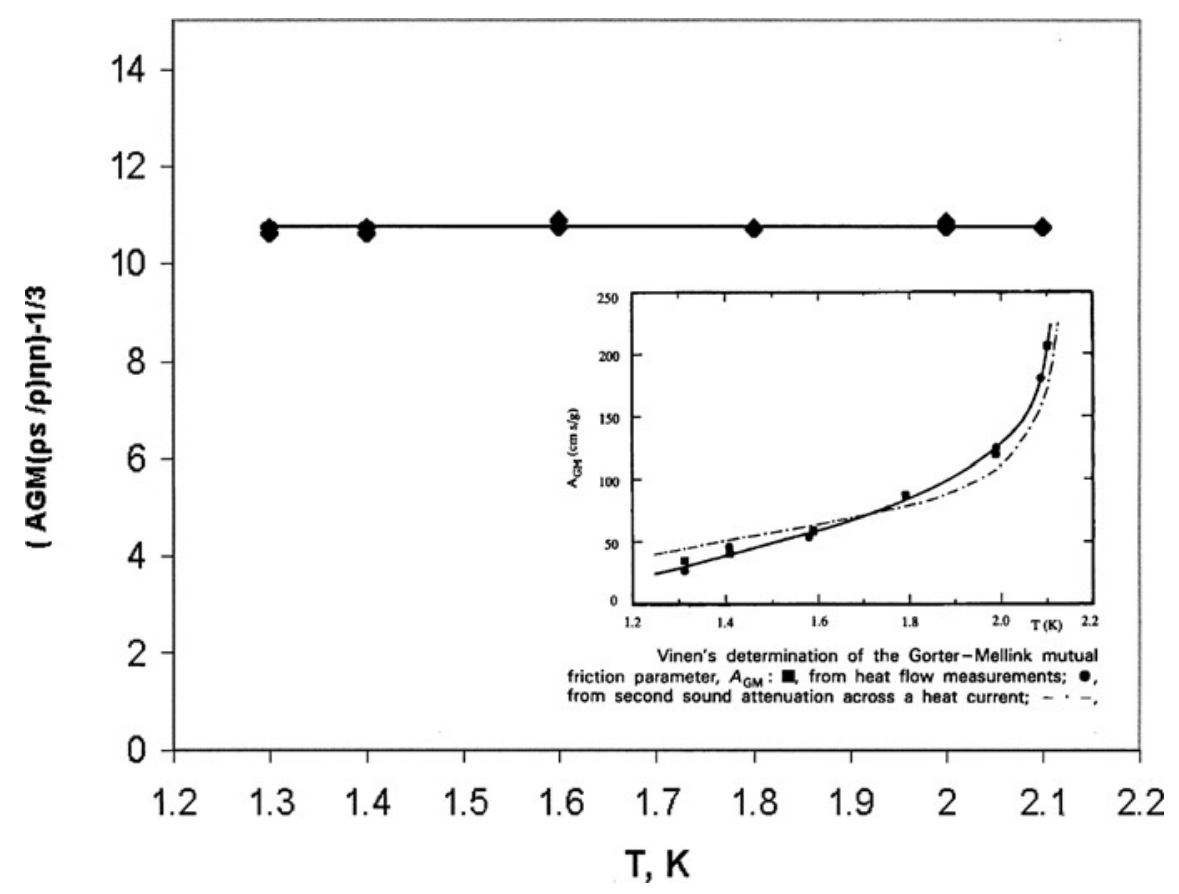

FIGURE 3

$K_{\mathrm{GM}}=\left(A_{\mathrm{GM}}\left(\rho_{s} / \rho\right) \eta_{n}\right)^{-1 / 3}$ versus the He II temperature based on data from the Gorter-Mellink constants measured by Vinen $(1957,1958)$. The solid line represents $K_{\mathrm{GM}}=11.3$. The insert shows a good fit of the equation with the $A_{\mathrm{GM}}$ test data. 


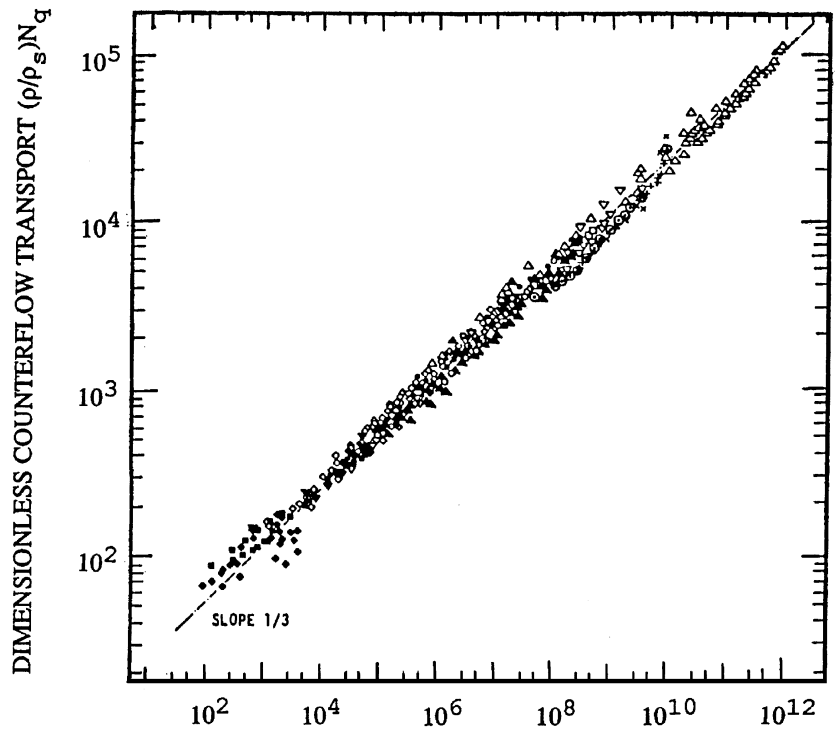

DIMENSIONLESS THERMOMECHANICAL DRIVING FORCE $\left(\rho_{s} / \rho_{n}\right) N_{\nabla T}$

FIGURE 4

Dimensionless turbulent He II heat transport in wide capillaries. The solid line represents Equation (21), $K_{\mathrm{GM}}=$ 11.3 .

Attempts have been made to curve-fit all thermodynamic properties in Equation (22) as a function of temperature and pressure:

$$
q=\left[f^{-1}(T, P) \nabla T\right]^{1 / 3}
$$

where

$$
f^{-1}(T, P)=\frac{\rho^{2} S_{\lambda}^{4} T^{3}}{A c}\left\{\left(\frac{T-\varsigma}{T_{\lambda}}\right)^{5.7}\left[1-\left(\frac{T-\varsigma}{T_{\lambda}}\right)^{5.7}\right]\right\}^{3} .
$$

It was found that using $(T-\zeta) / T_{\lambda}$ instead of $T / T_{\lambda}$ in the above equation fits the experimental data better. Properties at the $\lambda$ point for a given pressure are used for the calculation. For example, $S=1559 \mathrm{~J} / \mathrm{Kg}-\mathrm{K} ; A c=1150 \mathrm{~m}-\mathrm{s} / \mathrm{Kg}$; and $T=2.172 \mathrm{~K}$ for saturated He II. The $T=2.163 \mathrm{~K}$ is taken for He II under atmospheric pressure. The values of $A_{c}$ and $\zeta$ are given in the Table 1. Equation (24) is compared with experimental data collected by Srinivasan and Hofmann (1985) for various pressures. Figure 5 shows the curve-fit results. Baudouy and colleagues (2000) showed the results of cooling superconductor cables in superfluid, which demonstrated the transition between the laminar and turbulent transport regimes. The transport results are in good agreement with the formula presented above.

\section{Force Convection Superfluid Flow}

The heat transports discussed earlier are for zero net mass flow conditions. Superfluid He II can be forced through narrow
TABLE 1

Values of $A_{c}$ and $\zeta$ in Equation (24)

\begin{tabular}{lcc}
\hline$P(\mathrm{Mpa})$ & $A_{c}(\mathrm{~m}-\mathrm{s} / \mathrm{Kg})$ & $\zeta(\mathrm{K})$ \\
\hline Saturated vapor pressure & 1150 & 0 \\
0.1 & 1265 & 0 \\
0.25 & 1388 & 0 \\
0.5 & 1640 & .01 \\
0.7 & 1790 & .01 \\
1.0 & 2817 & .01 \\
1.5 & 4428 & .01 \\
2.0 & 7749 & .01 \\
2.5 & 13776 & .01 \\
\hline
\end{tabular}

restriction isothermally by gravitational force, fountain pressure, or plunger. Because the fluid flow is driven by the pressure difference instead of thermal mechanical forces, a new driving force number has to be introduced: $N_{\nabla P}=\rho \nabla P L_{c}^{3} / \eta_{n}^{2}$. Vote and colleagues (1971) and Frederking and colleagues (1971) proposed the resistance ratio $R / R_{N}$, which is the ratio of flow resistance, $R=\nabla P / v$, to its laminar value, $R_{N}$. For Newtonian flow, the laminar regime gives $R / R_{N}=N_{\nabla P} / N_{q}=1$. Superfluid is a non-Newtonian fluid, which presents unique behaviors as compared with classic fluid. It is appropriate to relate the resistance ratio and the driving forced by a superfluid fluid density ratio $\rho_{s} / \rho$. According to the power law, the superfluid resistance ratio can be written as

$$
\left(R / R_{N}\right) \rho_{s} / \rho=C_{n} N_{\nabla P}^{n} .
$$

Figure 6 shows the relationship between the dimensionless resistance ratios and the dimension less driving force. Table 2 summarizes the several He II transport regimes. The $n$ and $C_{n}$ numbers, and the relationship between the flow velocity and the $N_{\nabla P}$ for the Ladau flow regime to the Blasius flow regimes, as described by Schlichting (1960), are also tabulated.

\section{He II TRANSIENT HEAT AND MASS TRANSFER}

\section{Transient Transport of Surface Heat}

Various transient heat-transfer mechanisms were visually observed by Katsuki and colleagues (1995). After the stepwise heating of a flat plate heater in He II, local boiling sites appear and develop, eventually covering the heater surface, followed by the onset of film boiling. The state may further proceed to noisy boiling if the hydrostatic pressure is sufficiently great. It was found that there are some distinctions between cases with large and small $q$ values in the functional relationships of the onset time on $q$. At smaller $q$, the relationship follows $t_{p} \sim q^{-1 / 4}$. For larger $q$, the relationship changes to $t_{p} \sim q^{-1 / 2}$.

These relationships are analogous to those found for transient heat transfer in He II-filled ducts, which will be described later. It appears that the flow analysis in the duct is a one-dimensional analysis. The transient surface heat transport is considered as 


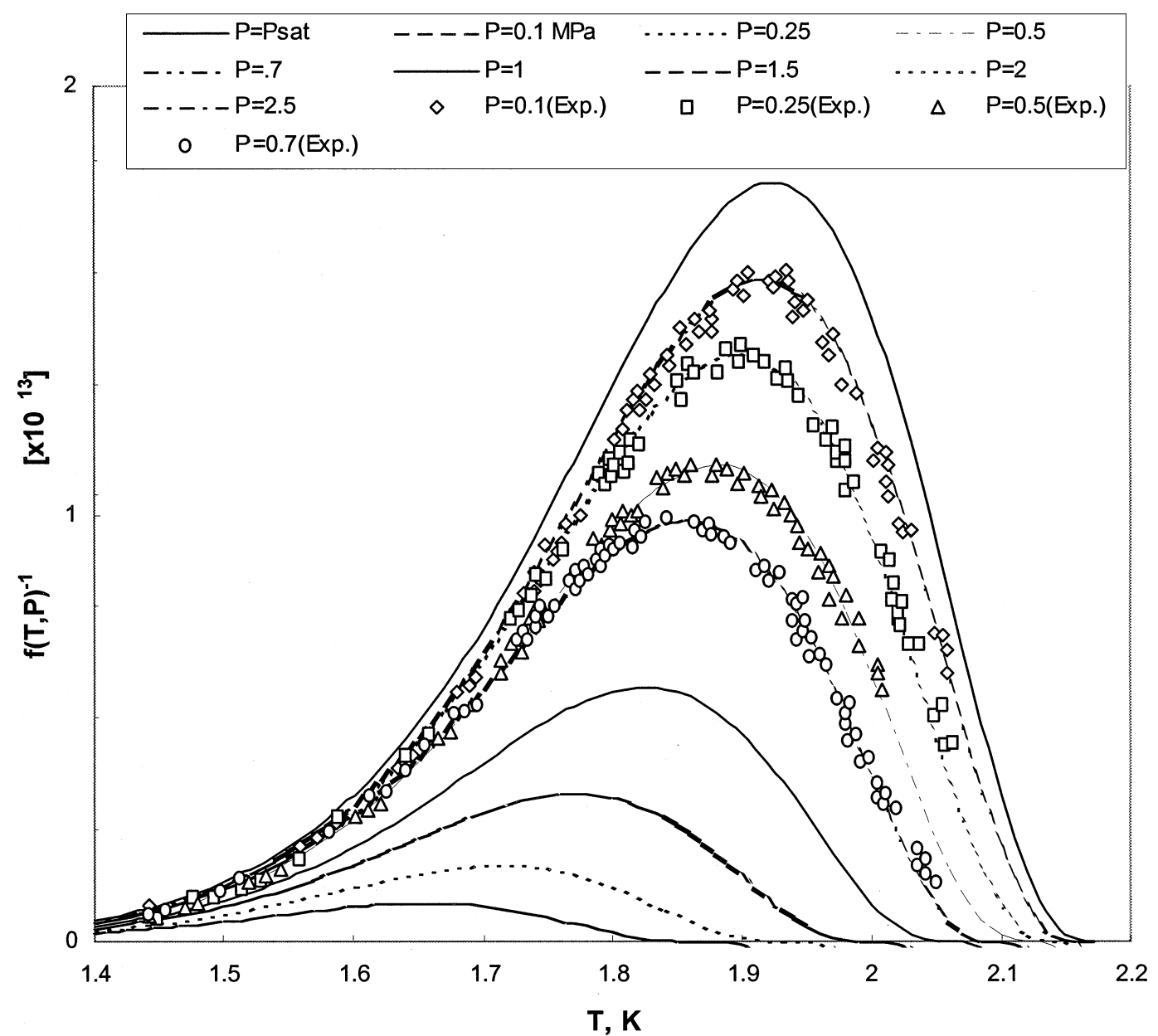

FIGURE 5

The heat conduction function versus temperature and pressure. The unit of the pressure is Mpa.

a three-dimensional transport. Apparently, the analytical results obtained from the one-dimensional case can qualitatively describe the case of three-dimensional surface heat transport. Many data taken from Katsuki and colleagues (1995), Stamm (1992), and Tsoi and Lutset (1986) for flat-plate geometry support these relationships. Wang and Zhang (1996) measured transients from thin wires to a bath of subcooled He II, which also showed similar relationships. Figure 7 shows the relationship between the peak heat-flux density and the onset time of film boiling for several surface transport geometries.

\section{Transient Transport of Heat Through Ducts}

A special case of transient entropy transport and heat transfer is considered; it combines relatively slow quasisteady equilibration with constraints given by the first law of thermodynamics.

TABLE 2

Characteristics of the Superfluid Flow

\begin{tabular}{lcccc}
\hline Flow Regime & $N_{\nabla P}$ range & $n$ & $C_{n}$ & $\begin{array}{c}\text { Velocity } \\
\text { Dependency }\end{array}$ \\
\hline Landau loss-less & $10^{-4}$ to $10^{-2}$ & 1 & $0.01 \sim 0.02$ & $v \sim N_{\nabla P} 0$ \\
Size-dependant & $10^{-2}$ to 1 & $3 / 4$ & $0.025 \sim 0.05$ & $v \sim N_{\nabla P} 1 / 4$ \\
Gorter-Mellink alike turbulent & 1 to 100 & $2 / 3$ & $0.016 \sim 0.064$ & $v \sim N_{\nabla P} 1 / 3$ \\
Blasius fully developed turbulent & 100 to 10000 & $3 / 7$ & $\sim 0.1$ & $v \sim N_{\nabla P} 1.75$ \\
\hline
\end{tabular}




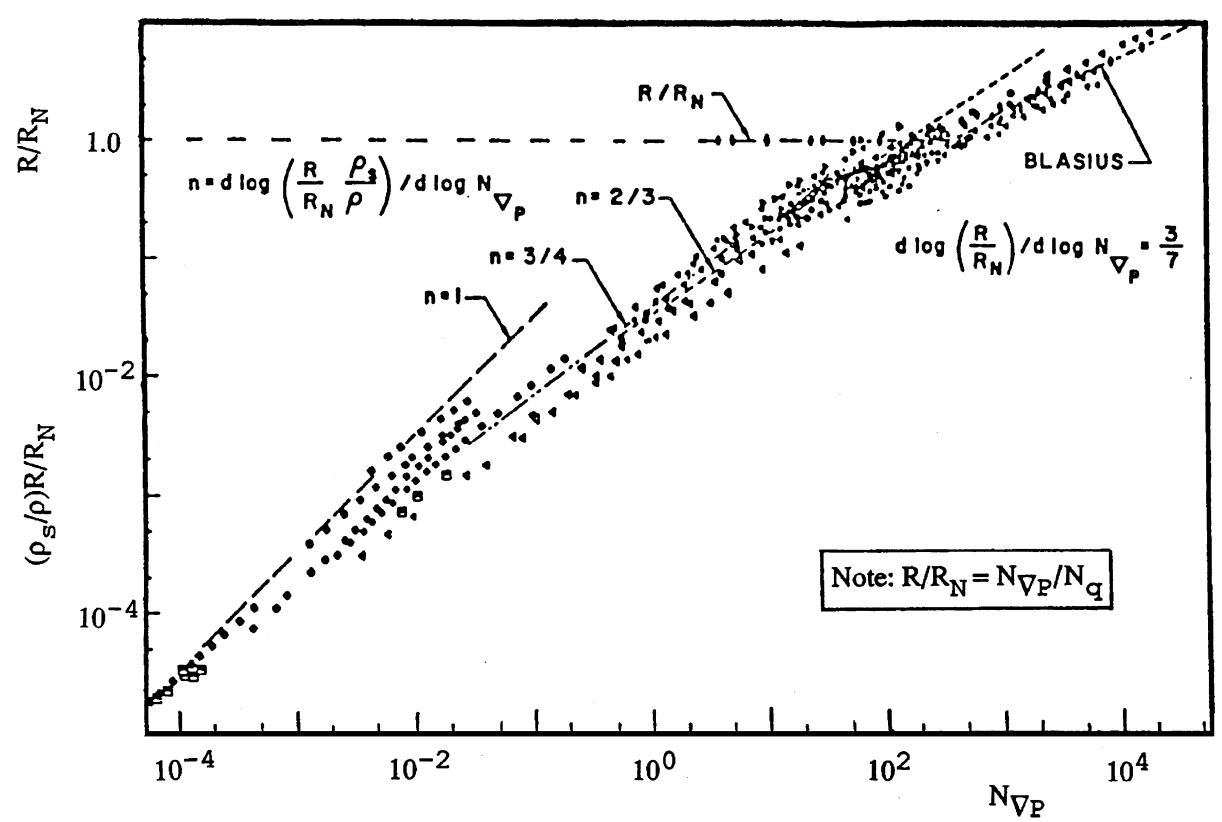

FIGURE 6

Superfluid transport in narrow capillaries (From Vote, 1965; Vote et al., 1971).

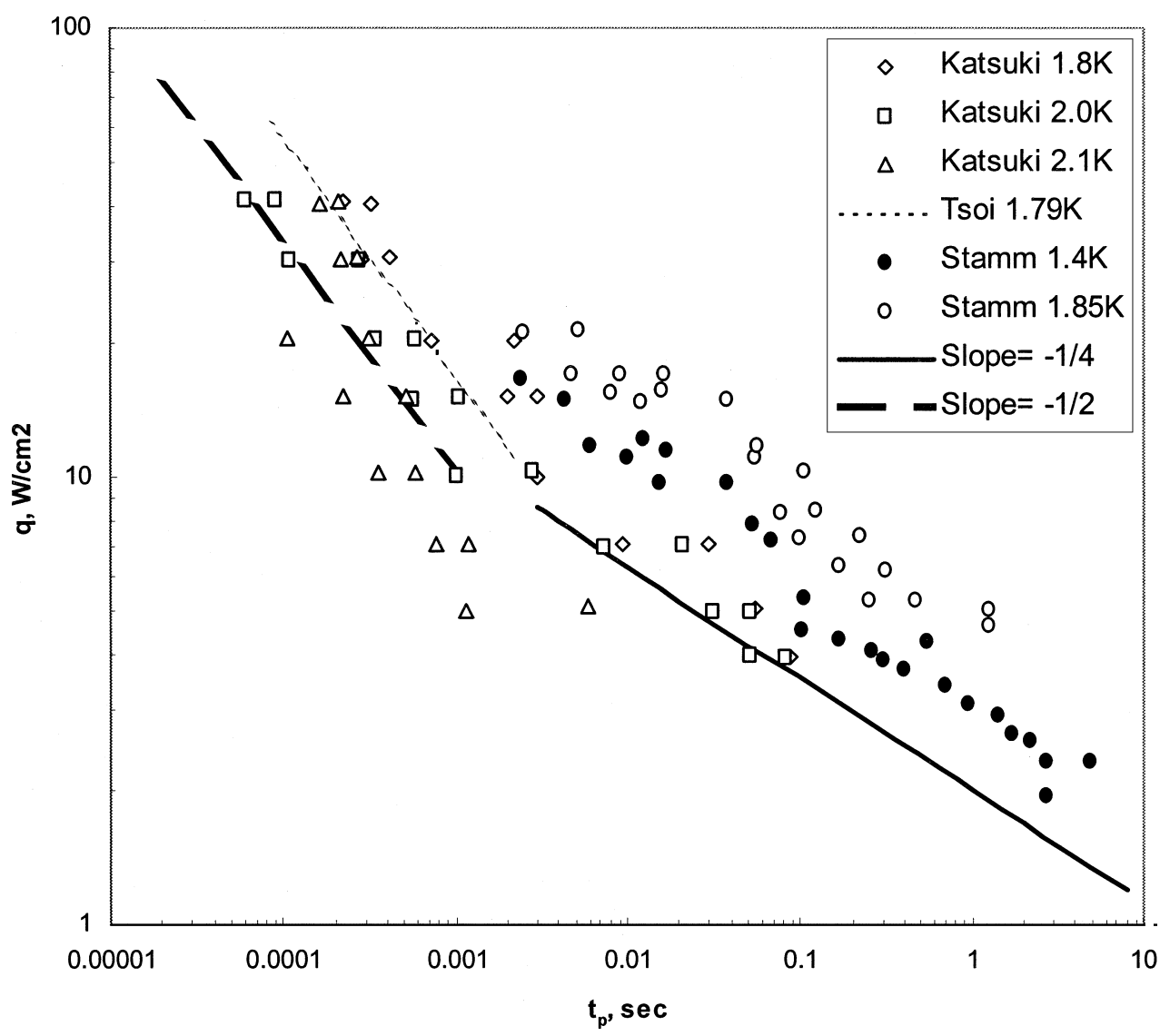

FIGURE 7

Transient surface-heat transfer, onset of film boiling. The solid lines indicate that the slope is approaching $-\frac{1}{2}$ for larger heat inputs, and it is approaching $-\frac{1}{4}$ for smaller heat inputs. (Data points from Katsuki et al., 1995; Tsoi and Lutset, 1986; 
According to the latter, for zero shaft power in an energy exchange system, the enthalpy change in unit time has to be equal to the heat supply rate to the volume subjected to heating. To simulate a sudden localized heating in a superconductor winding, one can construct a simple heat-transport model. It is assumed that sudden heat is generated at the bottom of a duct with adiabatic walls, a Gorter-Mellink duct resulting from external application of step heat-flux input.

In a Gorter-Mellink duct with a constant cross-section $A_{t}$, the combined first and second law is written for the heated section $A_{t} \cdot q \cdot d t=d H_{\text {tot }} ; d H_{\text {tot }}$ is the enthalpy, which varies with the temperature. The temperature is expressed in terms of a local function $T=T(x)$. After integration for a constant $q$ value, the thermal energy supplied in time $t$ is $q \cdot t=L_{R} \cdot \kappa \cdot \rho \cdot C_{p} \cdot \Delta T$, where $\Delta T \ll T . L_{R}$ is a reference length, which is a constant since most of the heat is generated at the vicinity of the heater surface, and $C_{p}$ is the specific heat at a constant pressure.

In general, the specific heat varies with temperature. Therefore, a thermophysical property integral has to be evaluated:

$$
q \cdot t=\kappa \cdot L_{R} \int_{T}^{T+\Delta T} \rho \cdot C_{p} \cdot d T .
$$

According to the Gorter-Mellink equation for steady heat transport in the laminar flow regime, Equation (16), the duct length is related to the thermophysical property integral:

$$
L=q^{-1} \int_{T}^{T+\Delta T} \frac{\rho^{2} S^{2} L_{c}^{2} T}{\eta_{n}} d T .
$$

The heated section is a fraction of the entire duct length, or $L_{R}=\xi L$. Equations (26) and (27) can be combined:

$$
t=\frac{\kappa \xi}{q^{2}} \int_{T}^{T+\Delta T} \rho C_{p} d T \int_{T}^{T+\Delta T} \frac{\rho^{2} S^{2} L_{c}^{2} T}{\eta_{n}} d T .
$$

For very small temperature differences in the duct, a simplified form can be used:

$$
t=\kappa \cdot \xi \cdot q^{-2} \cdot\left(\rho / \eta_{n}\right) \cdot C p \cdot\left(\rho \cdot S \cdot L_{c} \cdot T\right)^{2} \cdot(\Delta T / T)^{2} \cdot T .
$$

It becomes evident that the amount of time required to establish a specific temperature difference in a Gorter-Mellink duct, when the flow is in the laminar flow regime, is inversely proportional to the square of the input heat-flux density.

The same approach can be used to evaluate the relationship between the transient time and the heat flux for the turbulent regime. The duct length is then related to Equation (21):

$$
L=K_{\mathrm{GM}}^{3} q^{-3} \cdot \int_{T}^{T+\Delta T} \frac{\eta_{n} S^{4} \rho_{s}^{4} T^{3}}{\rho \rho_{n}} d T .
$$

Again assuming that $L_{R}=\xi L$, to get the transient time

$$
t=\frac{\kappa \cdot \xi \cdot K_{\mathrm{GM}}^{3}}{q^{4}} \cdot \int_{T}^{T+\Delta T} \rho C_{p} d T \cdot \int_{T}^{T+\Delta T} \frac{\eta_{n} S^{4} \rho_{s}^{4} T^{3}}{\rho \rho_{n}} d T .
$$

For very small temperature differences, the above equation can be reduced to the more simplified form:

$$
t=\zeta \cdot K_{\mathrm{GM}}^{3} \cdot q^{-4} \cdot\left(\frac{\eta_{n}}{\rho_{n}}\right) \cdot C_{p}\left(\rho_{s} S T\right)^{4}(\Delta T / T)^{2} T
$$

where $\zeta=\kappa \cdot \xi$, which is a constant. It is appropriate to introduce a dimensionless form on the basis of a heat ratio $N_{q *}$ and a dimensionless time, the Fourier number $N_{t}$ :

$$
N_{q_{*}}=q / q_{R} ; \quad q_{R}=\rho_{s} S T\left\{\frac{\eta_{n} C_{p} \Delta T^{2}}{\rho L_{c}^{2} \rho_{n} T}\right\}^{1 / 4}
$$

and

$$
N_{t}=t / t_{R} ; \quad t_{R}=\rho L_{c}^{2} / \eta_{n}
$$

Equation (32) is simplified to

$$
N_{q *}=\left(\zeta K_{\mathrm{GM}}^{3}\right)^{1 / 4}(N)_{t}^{-1 / 4} .
$$

The product $q \cdot t^{1 / 4}$ does not depend on a reference length $L_{R}$, and it is influenced only by the properties and the temperature difference in the duct. Figure 8 shows the transition from the transient laminar flow and the transient turbulent regime. The data taken from Chuang (1981) shows good agreement with the dimensionless analysis.

Figure 9 shows the dimensionless analysis of the data generated by Van Sciver (1979) The thermophysical properties are taken an arithmetic mean temperature of the He II domain heated up during transient heat transfer. It is seen that the data for the

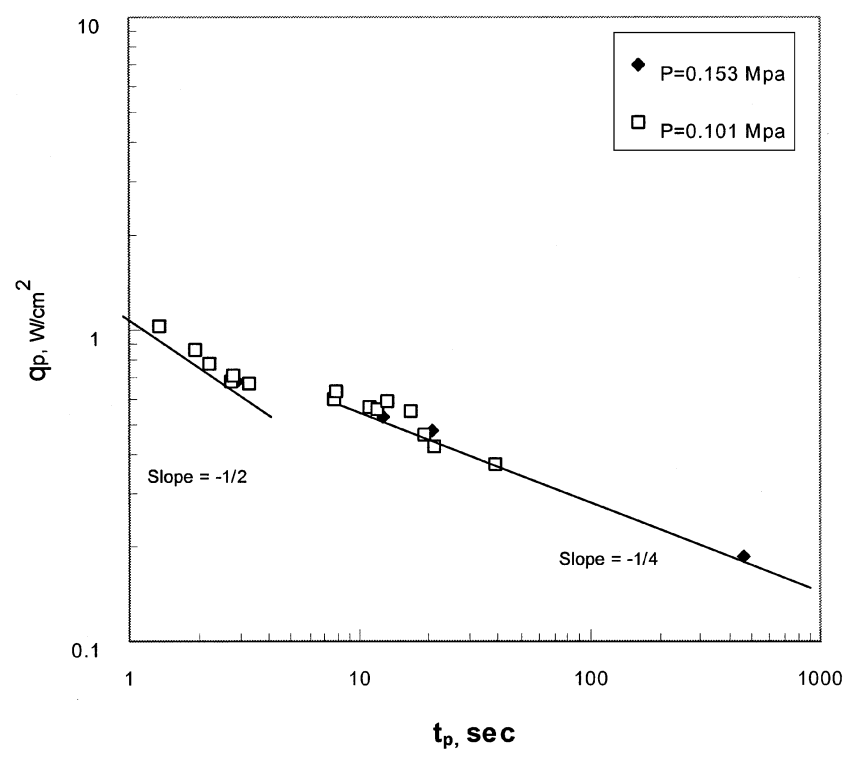

FIGURE 8

Transient peak heat-flux density versus time (bath temperature $=2.05 \mathrm{k}$ ). The solid lines represent slopes of $-\frac{1}{2}$ and $-\frac{1}{4}$. (Data points from Chuang, 1981). 


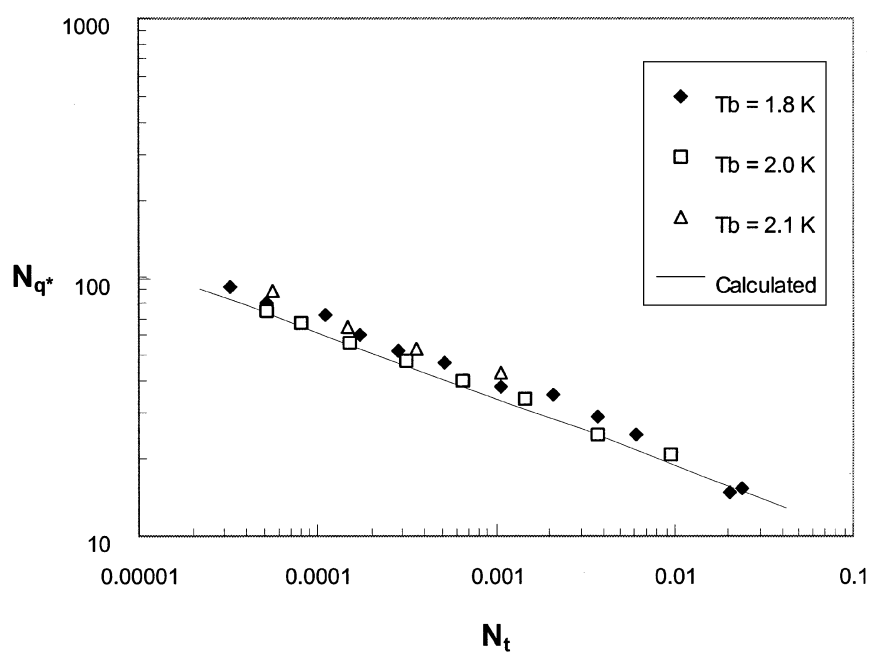

FIGURE 9

Dimensionless heat-flux density versus dimensionless time for transient Gorter-Mellink flow. Solid line slope $=-\frac{1}{4} ; K_{\mathrm{GM}}=$ $11.3, \zeta=1$ as compared to Equation (35). (Data from Van Sciver, 1979).

bath temperatures $2.11 \mathrm{~K}, 2.00 \mathrm{~K}$, and $1.8 \mathrm{~K}$ are described well by Equation (35). The value of $\left(\zeta K_{\mathrm{GM}}^{3}\right)^{1 / 4}$ is equal to 16.1 .

Again, it becomes evident that the amount of time required to establish a specific temperature difference in a Gorter-Mellink duct, when the flow is in the turbulent-flow regime, is inversely proportional to the fourth power of the input heat-flux density.

\section{CONCLUSIONS}

Favorable steady and transient heat-transport characteristics of superfluid as a coolant for superconducting rotating machines have been presented. This design formula can be used to effectively enhance the reliability and the performance of superconducting machines. Steady and transient superfluid transport in various transport modes (laminar, turbulent convection, fully developed turbulent, and forced flow) and geometry are characterized within a common frame of reference by the introduction of dimensionless numbers. Experimental data are then compiled to substantiate the analysis.

For steady-state heat transport, Kapitza conductance dominates the heat transport for surface-heater geometry. The Kapitza conductance is a function of the material and the surface treatment. Heat transport in ducts filled with superfluid can experience a laminar convection regime, turbulent convection, and fully developed transport regimes as the heat-flux density is raised. The heat transport in these regimes can be described by a universal dimensionless relationship.

For transient transport, similar characteristics were observed in surface heat transfer and heat transport in duct geometry. The dimensionless relationships derived for steady-state transport were extended to transient transport. A dimensionless relation- ship between the heat-flux density and the film boiling onset time was also developed.

\section{NOMENCLATURE}

$A_{t} \quad$ Cross-sectional area $\left(\mathrm{m}^{2}\right)$

$A_{\mathrm{GM}} \quad$ Gorter-Mellink parameter $(\mathrm{m} \cdot \mathrm{sec} / \mathrm{Kg})$

$C \quad$ Specific heat $(\mathrm{J} / \mathrm{kg} \cdot \mathrm{K})$

$D \quad$ Diameter (m)

$f(T)^{-1}$ Turbulent superfluid heat conductivity function $\left(W^{3} / \mathrm{m}^{5} \cdot \mathrm{K}\right)$

$F_{s n} \quad$ Mutual friction $(\mathrm{Pa} / \mathrm{m})$

$h \quad$ Heat transfer coefficient $\left(\mathrm{W} / \mathrm{m}^{2} \cdot \mathrm{K}\right)$

$H \quad$ Enthalpy (J)

$K_{\mathrm{GM}} \quad$ Gorter-Mellink constant

$L \quad$ Length (m)

$N \quad$ Dimensionless number

$P \quad$ Pressure $(\mathrm{Pa})$

$q \quad$ Heat flux density $\left(\mathrm{W} / \mathrm{m}^{2}\right)$

$Q \quad$ Heat flux (W)

$R / R_{N} \quad$ Resistance ratio

$S \quad$ Entropy $(\mathrm{J} / \mathrm{Kg} \cdot \mathrm{K})$

$t \quad$ Time (sec)

$T \quad$ Temperature (K)

$v \quad$ Velocity $(\mathrm{m} / \mathrm{sec})$

\section{Greek \\ $\rho \quad$ Density $\left(\mathrm{kg} / \mathrm{m}^{3}\right)$ \\ $\eta \quad$ Viscosity $\left(\mathrm{N} \cdot \mathrm{sec} / \mathrm{m}^{2}\right)$ \\ $\xi \quad$ Constant \\ $\zeta \quad$ Constant \\ $\kappa \quad$ Constant \\ $\sigma \quad$ Constant \\ $\nabla \quad$ Gradient}

$\begin{array}{ll}\text { Subscripts } \\ b & \text { Bath } \\ c & \text { Characteristic } \\ k & \text { Kapitza } \\ n & \text { Normal } \\ p & \text { Peak } \\ R & \text { Reference } \\ s & \text { Superfluid } \\ \text { sat } & \text { Saturated vapor pressure } \\ \lambda & \text { At lambda temperature } \\ \text { GM } & \text { Gorter-Mellink } \\ q & \text { Heat flux density } \\ \nabla T & \text { Temperature gradient driving force } \\ q^{*} & \text { Transient heat flux density } \\ t & \text { Time }\end{array}$

\section{REFERENCES}

Aized, D. 1999. Status of the 1,000 HP HTS motor development. IEEE Transactions AS-9:1197-1200. 
Boudouy, B., Francois, M. X., Juster, F. P., and Meuris, C. 2000. He II heat transfer through superconducting cables electrical insulation. Cryogenics 40:127.

Brechna, H. 1973. Superconducting Magnet Systems. New York: Springer-Verlag.

Chuang, T. C. 1981. Transients in He II due to Dynamic Quench of Superconductor. (PhD diss, University of California, Los Angeles).

Gorter, C. J., and Mellink, J. H. 1949. On the irreversible processes in liquid helium II. Physica 15:285.

Hull, J. R., Gupta, S. S., and Gaines, J. R. 1999. Trapped-flux internal-dipole superconducting motor/generator. IEEE Transaction AS-9:1229-1232.

Iwamoto, A., Maekawa, R., and Mito, T. 2001. Kapitza conductance of an oxidized copper surface in saturated He II. Cryogenics 41:367.

Katsuki, Y., Murakami, M., Lida, T., and Shimazaki, T. 1995. Visualization study of film boiling onset and transition to noisy film boiling in He II. Cryogenics 35:631.

Khalatnikov, I. M. 1952. Heat exchange between a solid body and $\mathrm{He}$ II. Journal of Experimental Theoretical Physics USSR 22:687.

Kovalev, L. K., et al. 1999. Alternators that use HTSC wire coils and bulk YBCO materials. Proceedings of the ICEC 17:379-382.

Leupold, M. J., Weggel, R. J., and Iwasa, Y. 1988. IEEE Transactions on Magnetics 24:1070.

Miller, J. R., Bird, M. D., Bole, S., Bonito-Oliva, A., Eyssa, Y., Kenney, W. J., Painter, T. A., Schneider-Muntau, H.-J., Summers, L. T., van Sciver, S. W., Welton, S., Wood, R. J., Williams, J. E. C., Bobrov, S., Iwasa, Y., Leupold, M., Stejskal, V., and Weggel, R. 1994. IEEE Transactions on Magnetics 30:1563.

Schlichting, H. 1960. Boundary Layer Theory, 4th ed, 502. New York: McGraw-Hill.

Shiotsu, M., Hata, K., Takeuchi, Y., Hama, K., and Sakurai, A. 1996. Estimation of Kapitza conductance effect on steady and transient boiling heat transfer in He I based on Kapitza conductance results in He II. Cryogenics 36:197-202.

Singh, S. K., Scherbarth, D. W., Ortoli, E. S., Repp, J. R., Christianson, O. R., Parker, J. H., Carr, J. W., and Gamble, B. 1999. Concep- tual design of a high-temperature superconducting generator. IEEE Transactions AS-9:1237-1240.

Soloski, S. C., and Frederking, T. H. K. 1980. International Journal of Heat Mass Transfer 23:437.

Srinivasan, R., and Hofmann, A. 1985. Investigations on cooling with forced flow of He II Part 2. Cryogenics 25:652.

Stamm, G. 1992. Acoustic, video and thermoacoustic registration of boiling-up phenomena in He II. Cryogenics 32(Supp):305.

Tisza, L. 1940. Sur la théorie des liquides quantiques: Application à l'hélium liquide. I \& II. Journal of Physics and Radium:164.

Tsoi, A. N., and Lutset, M. O. 1986. Inzhenerno-Fizicheskii Zhurnal 51:5-9.

Turck, B. 1989. IEEE Transactions on Magnetics 25:1473.

Vajda, I., Gobl, N., Meerovich, V., and Sokolovsky, V. 1999. Requirements for the industrial application of superconducting rotating electrical machines. IEEE Transactions AS-9:1225-1228.

Van Sciver, S. W. 1979. Transient heat transport in He II. Cryogenics 19:385.

Vinen, W. F. 1957. Proceedings of the Royal Society of London A240:114, 128, 193, 242.

Vinen, W. F. 1958. Proceedings of the Royal Society of London A243:400.

Vote, F. C. 1965. (MSc thesis, University of California, Los Angeles). Vote, F. C., Myers, J. E., Chu, H. B., and Frederking, T. H. K. 1971. Advanced Cryogenic Engineering 16:393.

Wang, R., and Zhang, P. 1996. Transient heat transfer form thin wires to a bath of sub-cooled superfluid helium. Cryogenics 36:1.

Yuan, S. W. K., Lee, J. M., Caspi, S., Soloski, S. C., Vote, F. C., Maddpx, J. P., Amar, R. C., Linnet, C., Kamioka, Y., Kim, Y. I., Chen, W. E. W., Schweikle, W. A., Hepler, W. A., Khandar, P., Carandang, R., Lee, J. Y., Kamegawa, M., Chuang, T. C., Chang, Y. W., and Chapman, R. C. 1990. Superfluid transport and its applications in space. Cryogenics 30:22.

Yuan, S. W. K., and Frederking, T. H. K. 1989. Thermal contact domain resistance: Overall solid helium 4 Kapitza resistance. Cryogenics 29:587. 

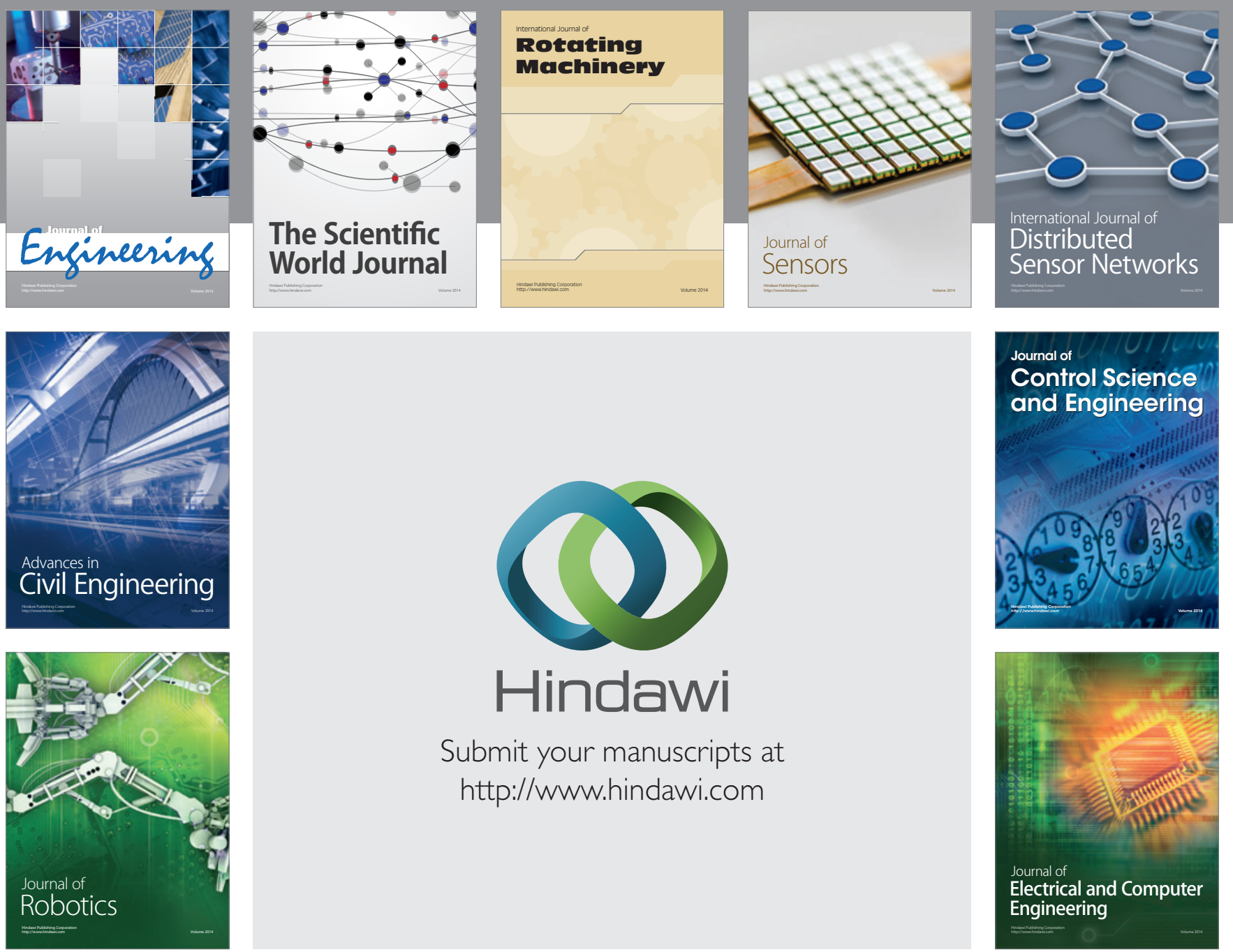

Submit your manuscripts at

http://www.hindawi.com
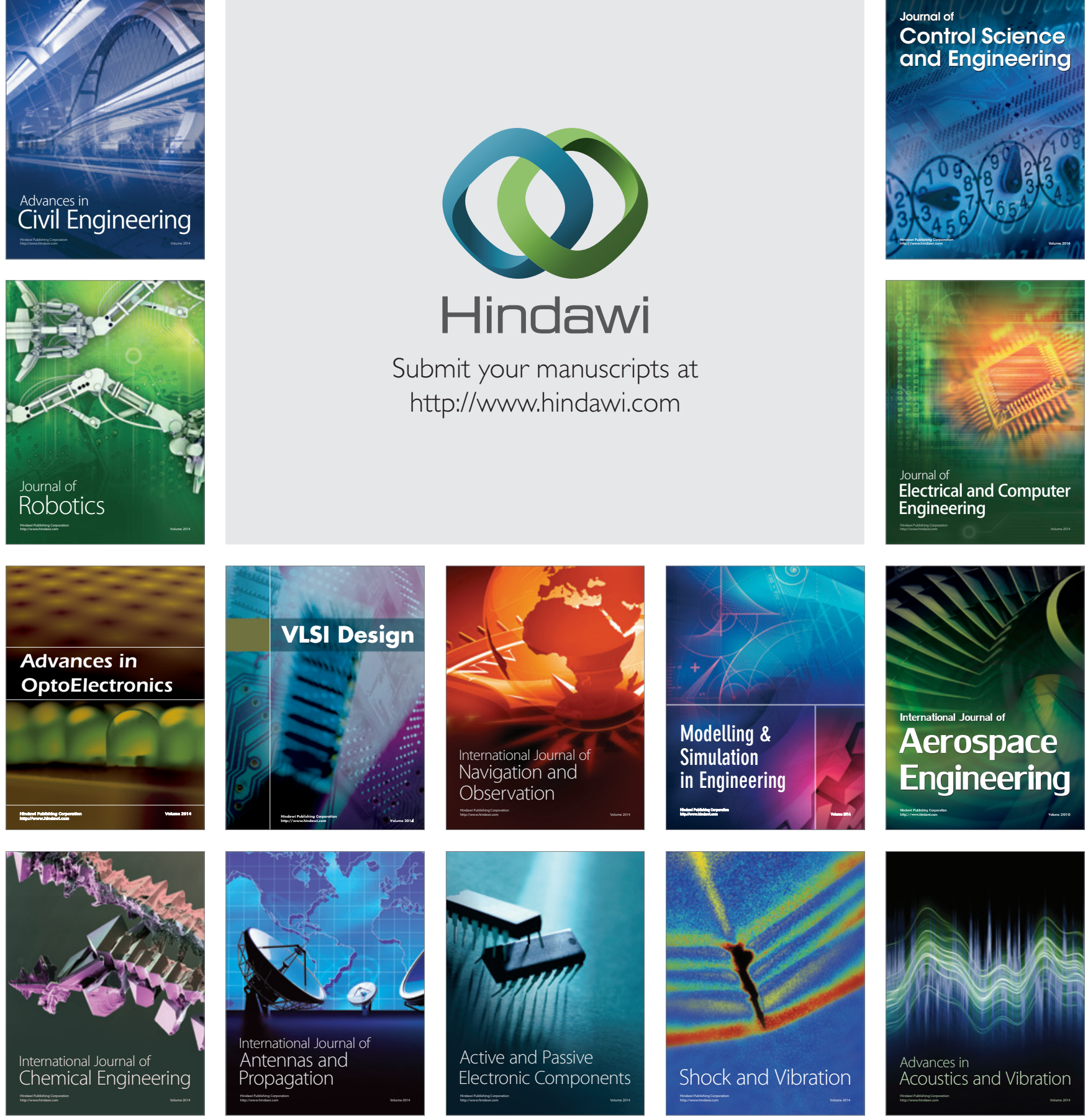